\title{
Infection management following ambulatory
}

\section{surgery}

This article was published in the following Dove Press journal:

Ambulatory Anesthesia

20 October 2015

Number of times this article has been viewed

\section{Anne B Chin \\ Elizabeth CWick}

Department of Surgery, The Johns Hopkins University School of Medicine, Baltimore, MD, USA
Correspondence: Elizabeth CWick The Johns Hopkins University School of Medicine, Blalock Room 658, 600 NWolfe St, Baltimore, MD 21287, USA

Email ewickI@jhmi.edu
Abstract: Surgical site infections (SSIs) are frequent postoperative complications that are linked to measures of surgical quality and payment determinations. As surgical procedures are increasingly performed in the ambulatory setting, management of SSIs must transition with this trend. Prevention of SSIs should include optimization of patient comorbidities, aggressive infection control policies including appropriate skin decontamination, maintenance of normothermia, and appropriate antibiotic prophylaxis. Systems must also be set in place to provide adequate surveillance for identification of SSIs when they do occur as well as provide direct feedback to surgeons regarding SSI rates. This may require utilization of claims-based surveillance. Patient education and close follow-up with the clinical team are essential for early identification and management of SSIs. Therapy should remain focused on source control and appropriate antibiotic therapy.

Keywords: ambulatory surgery, SSI, infection

\section{Introduction}

Surgical site infection (SSI) rate is one of the leading outcome measures of surgical quality, and is currently tied to payment determinations. ${ }^{1}$ SSIs are a common postoperative complication and account for approximately $20 \%$ of all hospital-acquired infections. ${ }^{2}$ The overall impact of these infections is undoubtedly larger than we can conclude based on these numbers alone, as SSIs are underreported and surveillance data is only available for limited procedures. It has been well established that SSIs following inpatient surgery result in increased morbidity, length of hospital stay, and cost. ${ }^{3,4}$

Currently, the majority of surgical procedures are performed in ambulatory settings, and the number increases each year. ${ }^{5,6}$ However, there is little data about SSIs following ambulatory surgery. In fact, the majority of ambulatory surgical centers (ASCs) are not required to report hospital-acquired infection to the National Health Safety Network (NHSN). Only facilities located within the states of Colorado, Massachusetts, Nevada, New Hampshire, New Jersey, and Texas are currently required to report SSIs to the NHSN. ${ }^{7}$

Inspections of Medicare-certified ASCs in 2008 revealed that lapses in infection control at these facilities were common, with $67.6 \%$ of ASCs having had at least one lapse in infection control during the study time period. ${ }^{8}$ Fortunately, despite the commonality of these breakdowns in protocol, reported rates of SSIs following ambulatory surgery, as assessed by administrative data, are generally low and comparable with rates reported for inpatient surgery. ${ }^{9-11}$ 


\section{Defining SSI}

The NHSN was established by the Centers for Disease Control and Prevention to monitor quality control measures including SSIs. As part of this monitoring process, the NHSN has developed a widely used set of criteria for defining SSIs. In this model, infections are categorized based on depth of involvement (Table 1). The categories are superficial incisional, deep incisional, and organ/space.

Most SSIs are the result of contamination either from the skin, the tissues surrounding the incision, or from other structures involved in the operative procedure. The most common pathogens associated with SSI are Staphylococcus aureus, coagulase-negative Staphylococci, Escherichia coli,
Enterococcus faecalis, and Pseudomonas aeruginosa according to the NHSN surveillance report from 2009 to $2010 . .^{12}$

\section{Risk factors}

There are a number of variables that influence the likelihood of developing an SSI. SSIs develop as the result of a complex interaction of variables ranging from type of procedure involved to patient-related factors.

\section{Procedure}

All surgical procedures carry a risk of infection. However, SSI rates can vary greatly between procedures. This is in part

Table I Surgical site infection criteria

\begin{tabular}{|c|c|}
\hline Category & Criteria \\
\hline \multirow[t]{13}{*}{ Superficial incisional SSI } & Infection occurs within 30 days after the operative procedure (where day I= the procedure date) \\
\hline & AND \\
\hline & Involves only skin and subcutaneous tissue of the incision \\
\hline & AND \\
\hline & Patient has at least one of the following: \\
\hline & a. purulent drainage from the superficial incision \\
\hline & b. organisms isolated from an aseptically obtained culture from the superficial incision or subcutaneous tissue \\
\hline & c. superficial incision that is deliberately opened by a surgeon, attending physician, or other designee and is culture \\
\hline & positive or not cultured \\
\hline & AND \\
\hline & Patient has at least one of the following signs or symptoms: pain or tenderness; localized swelling; erythema; or \\
\hline & heat. A culture negative finding does not meet this criterion \\
\hline & d. diagnosis of a superficial incisional SSI by the surgeon or attending physician or other designee \\
\hline \multirow[t]{13}{*}{ Deep incisional SSI } & Infection occurs within 30 or 90 days after the operative procedure (where day $I=$ the procedure date) \\
\hline & AND \\
\hline & Involves the deep soft tissues of the incision (eg, fascial and muscle layers) \\
\hline & AND \\
\hline & Patient has at least one of the following: \\
\hline & a. purulent drainage from the deep incision \\
\hline & b. a deep incision that spontaneously dehisces or is deliberately opened or aspirated by a surgeon, attending \\
\hline & physician or other designee and is culture positive or not cultured \\
\hline & AND \\
\hline & Patient has at least one of the following signs or symptoms: fever $\left(>38^{\circ} \mathrm{C}\right)$; localized pain or tenderness. \\
\hline & A culture negative finding does not meet this criterion \\
\hline & c. An abscess or other evidence of infection involving the deep incision that is detected on gross anatomical or \\
\hline & histophathologic exam or imaging test \\
\hline \multirow[t]{13}{*}{ Organ/space SSI } & Infection occurs within 30 or 90 days after the operative procedure (where day $I=$ the procedure date) \\
\hline & AND \\
\hline & Infection involves any part of the body deeper than the fascial/muscle layers, which is opened or manipulated during \\
\hline & the operative procedure \\
\hline & AND \\
\hline & Patient has at least one of the following: \\
\hline & a. purulent drainage from a drain that is placed into the organ/space (eg, closed suction drainage system, open drain, \\
\hline & T-tube drain, CT-guided drainage) \\
\hline & b. organisms isolated from an aseptically obtained culture of fluid or tissue in the organ/space \\
\hline & c. an abscess or other evidence of infection involving the organ/space that is detected on gross anatomical or \\
\hline & histopathologic exam, or imaging test \\
\hline & AND \\
\hline & Meets at least one criterion for a specific organ/space infection site \\
\hline
\end{tabular}

Note: Data from: Centers for Disease Control and Prevention (CDC). ${ }^{42}$

Abbreviations: SSI, surgical site infection; CT, computed tomography. 
due to variability in exposure to microbes during a procedure. For example, SSI rates have been reported as low as $0.5 \%$ in laparoscopic cholecystectomy to greater than $20 \%$ for colectomies. ${ }^{3,11,13,14}$

\section{Wound class}

Furthermore, the class of a surgical incision carries varying levels of risk for wound infection (Table 2).

Assessment of the National Nosocomial Infections Surveillance (NNIS) System hospitals data, the predecessor to NHSN, by Culver et al demonstrated increasing risk of infection with each wound class. ${ }^{15}$ This increased risk associated with wound class is included in the NNIS risk index for predicting SSI risk. This index was developed based on data obtained from the NNIS system hospitals and is measured on a scale of $0-3 .{ }^{15}$ The components of the index are 1) American Society of Anesthesiologists preoperative assessment score of 3,4 , or 5; 2 ) an operation with a wound classified as contaminated or dirty; and 3) an operation lasting longer than the 75 th percentile in duration for the specific procedure. ${ }^{15,16}$ Each factor included in the index contributes one point to the risk index score.

Table 2 Classification of wounds

\begin{tabular}{|c|c|}
\hline Category & Definition \\
\hline Clean & $\begin{array}{l}\text { An uninfected operative wound in which no } \\
\text { inflammation is encountered, and the respiratory, } \\
\text { alimentary, genital, or uninfected urinary tracts } \\
\text { are not entered. In addition, clean wounds are } \\
\text { primarily closed and, if necessary, drained with } \\
\text { closed drainage. Operative incisional wounds that } \\
\text { follow nonpenetrating (blunt) trauma should be } \\
\text { included in this category if they meet the criteria }\end{array}$ \\
\hline $\begin{array}{l}\text { Clean- } \\
\text { contaminated }\end{array}$ & $\begin{array}{l}\text { Operative wounds in which the respiratory, } \\
\text { alimentary, genital, or urinary tracts are entered } \\
\text { under controlled conditions and without unusual } \\
\text { contamination. Specifically, operations involving the } \\
\text { biliary tract, appendix, vagina, and oropharynx are } \\
\text { included in this category, provided no evidence of } \\
\text { infection or major break in technique is encountered }\end{array}$ \\
\hline Contaminated & $\begin{array}{l}\text { Open, fresh, accidental wounds. In addition, } \\
\text { operations with major breaks in sterile technique } \\
\text { (eg, open cardiac massage) or gross spillage from the } \\
\text { gastrointestinal tract and incisions in which acute, } \\
\text { nonpurulent inflammation is encountered including } \\
\text { necrotic tissue without evidence of purulent drainage } \\
\text { (eg, dry gangrene) are included in this category }\end{array}$ \\
\hline Dirty or infected & $\begin{array}{l}\text { Includes old traumatic wounds with retained } \\
\text { devitalized tissue and those that involve existing } \\
\text { clinical infection or perforated viscera. This } \\
\text { definition suggests that the organisms causing } \\
\text { postoperative infection were present in the } \\
\text { operative field before the operation }\end{array}$ \\
\hline
\end{tabular}

Note: Data from: Centers for Disease Control and Prevention (CDC). ${ }^{42}$

\section{Patient risk factors}

Patient-related factors that have been associated with an increased risk of developing an SSI include age, obesity, smoking, diabetes mellitus, malnutrition, dyslipidemia, and immunosuppression. ${ }^{17}$ Optimization of these comorbidities is critical to reducing the incidence of SSI. For example, careful optimization of glucose control has been shown to reduce SSI rates. ${ }^{17}$ Recommendations for glycemic control include a reduction in $\mathrm{HgbA}_{1 \mathrm{c}}$ to $<7.0 \%$ in addition to a reduction in serum glucose levels. ${ }^{18}$ Studies have also shown that smoking cessation reduces wound-related complications with current recommendations for smoking cessation at least 30 days prior to an operation. ${ }^{17,19}$

\section{Perioperative prevention}

There are numerous preventative measures in the operative period designed to reduce SSI rates. These include skin decontamination, perioperative warming, and antimicrobial prophylaxis.

It has been long recommended that skin decontamination be performed with topical antiseptic agents to help prevent infection. ${ }^{17}$ Chlorhexidine- and iodophor-based agents are the two most common classes of topical antiseptics. There have been numerous studies comparing the efficacy of these two groups of agents, although there has not been definitive evidence of a clinically significant difference between the two agent groups. ${ }^{20,21}$ It is clear that an alcohol-based skin preparation is superior. The two most commonly available preparations are solutions of isopropyl alcohol combined with either chlorhexidine gluconate or iodine povacrylex.

Perioperative hypothermia has been correlated with an increased risk of SSI. ${ }^{17,22,23}$ This is attributed to a number of factors including increased total oxygen consumption and decreased peripheral perfusion, induced coagulopathy, and reduced immune response secondary to the hypothermia. ${ }^{24}$ Although recent studies have called policies of strict temperature regulation into question, showing no significant difference between continuous measures of intraoperative temperature and the incidence of SSI, extreme hypothermia is detrimental. ${ }^{25}$ It is best practice to monitor the patient's temperatures in the operating room and to maintain core temperature $>36^{\circ} \mathrm{C}$. For longer procedures, this may require use of a forced air warming device, but for shorter outpatient procedures, this is likely not necessary but providers should also be cognizant of trying to avoid having patients uncovered for any period.

Antibiotic prophylaxis is indicated when the risk of perioperative bacterial contamination is high, as in 
clean-contaminated or contaminated procedures. It is also indicated in patients at high risk for serious morbidity as a consequence of infection, such as those undergoing cardiothoracic or neurosurgery procedures. ${ }^{26}$ Postoperative complications of cardiothoracic and neurosurgical procedures such as endocarditis, mediastinitis, sternal osteomyelitis, or meningitis can be devastating and warrant antimicrobial prophylaxis. Additionally, patients can have increased morbidity and risk of infection due to insertion of prosthetic materials or other foreign bodies, and should therefore also receive prophylaxis. This includes hernia repair with mesh and any orthopedic procedure using internal fixation devices. Other common ambulatory procedures that require antibiotic prophylaxis include appendectomy, most procedures involving the urinary tract, and adenotonsillectomy.

Clinical trials have shown significant reduction in SSI rates for moderate-to-high risk procedures with use of routine antibiotic prophylaxis. ${ }^{27,28}$ For low-risk procedures, such as clean operations without the use of prosthetic materials or other foreign bodies, the risks (adverse reactions) outweigh the potential benefit, and it is not recommended that they be administered. ${ }^{26}$ The basic principles of antibiotic prophylaxis are for the administration of a safe and appropriate antimicrobial agent administered in the preoperative period to allow serum and tissue concentrations to reach effective levels at the time of incision, and these antibiotics should be discontinued as soon as possible. ${ }^{17}$ In 2013, the American Society of Health-System Pharmacists, the Infectious Diseases Society of America, the Surgical Infection Society, and the Society for Healthcare Epidemiology of America released an update to clinical practice guidelines for antimicrobial prophylaxis. ${ }^{29}$ These guidelines recommend administration of antibiotic prophylaxis, if appropriate for the procedure, within 60 minutes of incision, or within 120 minutes for antibiotics requiring longer infusion times. ${ }^{29}$ In addition, weight-based dosing should be utilized to obtain appropriate levels of antibiotic in the obese patient. ${ }^{29}$ Another key component to maximizing effectiveness of antibiotics in reducing SSI is adequate redosing of antibiotics for longer operative procedures. It is recommended that antibiotics are redosed at intervals of two half-lives of the antibiotic used. ${ }^{29}$ This is supported by the TRAPE trial, which showed a reduction of SSI in the cohort who received antibiotic redosing. ${ }^{30}$

Recently, in attempts to further reduce SSIs, Schweizer et al implemented a bundled intervention of screening, decolonization, and targeted antimicrobial prophylaxis to prevent complex $S$. aureus in select cardiac and orthopedic patients. ${ }^{31}$ Patients were screened for colonization with MSSA or MRSA.
Those who were positive were treated with intranasal mupirocin and chlorhexidine bathing. MRSA carriers received vancomycin and cefazolin or cefuroxime for perioperative prophylaxis, while all others received cefazolin or cefuroxime alone. This intervention resulted in a decrease in mean SSI rate per 10,000 operations from 36 in the preintervention period to 21 in intervention period. They also noted a dose response association of bundle adherence and SSI rate reduction. ${ }^{31}$ There is potential for further reduction in SSIs by utilizing bundled tactics such as this in addition to other established techniques.

Another important component of preventing infection is proper decontamination of reusable surgical instruments. These instruments provide potential routes of transmission of pathogenic microorganisms between patients. Studies have shown lack of compliance with established guidelines for disinfection and sterilization and failures in decontamination have led to numerous outbreaks. ${ }^{32}$ The Centers for Disease Control and Prevention released guidelines for disinfection and sterilization in health care facilities in 2008. These guidelines should be used to direct sterile processing practices in surgical facilities. Per these guidelines, surgical instruments, cardiac and urinary catheters, implants, and ultrasound probes used in sterile body cavities are critical items that confer a high risk for infection if they are contaminated. As such, the majority of these items should be purchased sterile or sterilized with steam if possible. Heat-sensitive objects can be treated with ethylene oxide, hydrogen peroxide gas plasma, or by liquid chemical sterilants. Liquid chemical sterilants include $\geq 2.4 \%$ glutaraldehyde-based formulations, $0.95 \%$ glutaraldehyde with $1.64 \%$ phenol/phenate, $7.5 \%$ stabilized hydrogen peroxide, $7.35 \%$ hydrogen peroxide with $0.23 \%$ peracetic acid, $0.2 \%$ peracetic acid, and $0.08 \%$ peracetic acid with $1.0 \%$ hydrogen peroxide. Of note, liquid sterilants only produce sterility if cleaning precedes treatment and if proper guidelines for use are followed. ${ }^{33,34}$ All heat-sensitive endoscopes (gastrointestinal endoscopes, bronchoscopes, nasopharyngoscopes) must be properly cleaned and subjected to high-level disinfection after each use. This can be achieved using ethylene oxide sterilization or liquid chemical sterilants. ${ }^{33}$ Users should check with device manufacturers for specific information about germicide compatibility with their device, as there have been reported cosmetic and functional damage to endoscopes as a result of some of the chemicals in the liquid chemical sterilants.

\section{Surveillance}

Critical to management of SSIs in the setting of outpatient surgery is surveillance. Since these patients do not undergo 
a period of inpatient observation, the development of an SSI can be missed. Unfortunately, a reliable or standardized method for postdischarge surveillance has not yet been established. Research has shown that surveillance programs with feedback of SSI rates to surgeons can reduce subsequent rates by $30 \%-40 \%{ }^{35}$ Critical components of a successful surveillance program include intensive surveillance activities, infection control activities, and regular feedback of SSI rates to the surgeons. ${ }^{35}$

There are three main approaches to SSI surveillance: clinical registry with chart abstraction, administrative claims data, and patient-reported outcomes. Today, clinical registry-based surveillance is considered the gold standard. Comparison of registry data with administrative claims data for SSI surveillance has demonstrated that, for complex in patient procedures, registry data is far more sensitive and specific. ${ }^{36}$ This approach requires a large investment (data abstractors, program participation fees, etc); most hospitals limit this approach to high-risk procedures, especially those tied to reimbursement programs like cardiac, colorectal, or gynecologic surgery. It is rare for hospitals or ambulatory surgery centers to invest in this type of surveillance for outpatient procedures. An alternative approach that may decrease the resources needed for surveillance while maintaining data integrity is to monitor SSI rates through administrative claims-based SSI surveillance in conjunction with targeted medical record review. ${ }^{11,37,38}$ Claim codes from both inpatient and ambulatory encounters can be used to identify potential SSIs following ambulatory procedures. Hospitalbased surveillance methods alone are inadequate to monitor SSI following ambulatory surgery as many SSIs are managed purely in the outpatient setting. ${ }^{11}$ Furthermore, it is likely that patients who undergo surgery at a freestanding ambulatory center will be managed at another facility should they develop an SSI. Until we have true interoperability of the electronic health record, accurate SSI surveillance for procedures done in the outpatient setting is going to be challenging.

Close follow-up of patients for evidence of SSI should be undertaken in the ambulatory surgery population. Essential to the success of this process is patient education regarding the risks of developing SSIs, the methods to prevent SSIs, and the signs and symptoms of an SSI once it has developed. Ideally, SSI education should take place during the preoperative visit to allow the outpatient patient time to process the information appropriately. At discharge from surgery, patients should also receive additional information regarding SSI as a supplement to their preoperative education.

\section{Therapy}

Once an SSI has been identified, the basic principle of SSI therapy remains the same whether the patient has undergone inpatient or outpatient surgery. This principle is to control the infectious source.

Incision and drainage with local wound care are the standard management for a superficial incisional SSI. ${ }^{39,40}$ It is important that the wound is opened enough to allow for adequate drainage. Often, no antibiotic therapy is required for these infections. ${ }^{41}$ However, antibiotics should be considered in patients with systemic features or widening erythema. ${ }^{41}$

More complicated SSIs require antibiotic therapy in addition to source control. It is important that appropriate antibiotics are selected. Selection should be based on the expected microbial causes for infection. ${ }^{39,40}$

\section{Conclusion}

Surgical procedures are increasingly performed in ambulatory settings. The nature of outpatient surgery complicates the management of SSIs. Despite the fact that it has been established that SSIs are a common postoperative complication and constitute a large proportion of hospital-acquired infections in the inpatient population, there are limited data regarding SSIs in outpatient settings due to underreporting and difficulties with surveillance after surgery. However, what little data we have demonstrated compare SSI rates in ambulatory surgery with inpatient surgery.

Even with low SSI rates, the burden on the health care system is significant because of the large number of surgeries performed in the outpatient setting. It is critical to reduce SSI rates through optimization of patient factors and perioperative factors. Furthermore, improved surveillance of SSI in the outpatient setting is essential.

\section{Disclosure}

The authors report no conflicts of interest in this work.

\section{References}

1. Centers for Medicare and Medicaid Services (CMS). Medicaid program; payment adjustment for provider-preventable conditions including health care-acquired conditions. Final rule. Fedl Regist. 2011;76(108): 32816-32838.

2. Klevens RM, Edwards JR, Richards CL Jr, et al. Estimating health careassociated infections and deaths in US hospitals, 2002. Public Health Rep. 2007;122(2):160-166.

3. Lissovoy Gd, Fraeman K, Hutchins V, Murphy D, Song D, Vaughn BB Surgical site infection: incidence and impact on hospital utilization and treatment costs. Am J Infect Control. 2009;37(5):387-397.

4. Boltz MM, Hollenbeak CS, Julian KG, Ortenzi G, Dillon PW. Hospital costs associated with surgical site infections in general and vascular surgery patients. Surgery. 2011;150(5):934-942. 
5. Barie PS. Infection control practices in ambulatory surgical centers. JAMA. 2010;303(22):2295-2297.

6. National Center for Health Statistics. Health, United States, 2013: With Special Feature on Prescription Drugs. Hyattsville, MD: National Center for Health Statistics; 2013. Available from: http://www.cdc.gov/ nchs/data/hus/hus13.pdf. Accessed June 15, 2015.

7. Ambulatory Surgery Center Association. Revised Surgical Site Infection Surveillance Information. Alexandria, VA: Ambulatory Surgery Center Association; 2013; Available from: http://www.ascassociation.org/ aboutus/latestnews/newsarchive/2013/june2013/revisedsurgicalsitein fectionsurveillanceinformation/. Accessed June 15, 2015.

8. Schaefer MK, Jhung M, Dahl M, et al. Infection control assessment of ambulatory surgical centers. JAMA. 2010;303(22):2273-2279.

9. Owens PL, Barrett ML, Raetzman S, Maggard-Gibbons M, Steiner CA. Surgical site infections following ambulatory surgery procedures. JAMA. 2014;311(7):709-716.

10. Nazarian Mobin SS, Keyes GR, Singer R, Yates J, Thompson D. Infections in outpatient surgery. Clin Plast Surg. 2013;40(3):439-446.

11. Rhee C, Huang SS, Berrios-Torres SI, et al. Surgical site infection surveillance following ambulatory surgery. Infect Control Hosp Epidemiol. 2015;36(2):225-228.

12. Sievert DM, Ricks P, Edwards JR, et al. Antimicrobial-resistant pathogens associated with healthcare-associated infections: summary of data reported to the ational Healthcare Safety Network at the Centers for Disease Control and Prevention, 2009-2010. Infect Control Hosp Epidemiol. 2013;34(1):1-14.

13. Konishi T, Watanabe T, Kishimoto J, Nagawa H. Elective colon and rectal surgery differ in risk factors for wound infection: results of prospective surveillance. Ann Surg. 2006;244(5):758-763.

14. Smith RL, Bohl JK, McElearney ST, et al. Wound infection after elective colorectal resection. Ann Surg. 2004;239(5):599-605; discussion 605-597.

15. Culver DH, Horan TC, Gaynes RP, et al. Surgical wound infection rates by wound class, operative procedure, and patient risk index. National Nosocomial Infections Surveillance System. Am J Med. 1991;91(3B): $152 \mathrm{~S}-157 \mathrm{~S}$.

16. National Nosocomial Infections Surveillance System. National Nosocomial Infections Surveillance (NNIS) System Report, data summary from January 1992 through June 2004, issued October 2004. Am J Infect Control. 2004;32(8):470-485.

17. Mangram AJ, Horan TC, Pearson ML, Silver LC, Jarvis WR. Guideline for prevention of surgical site infection, 1999. Hospital Infection Control Practices Advisory Committee. Infect Control Hosp Epidemiol. 1999;20(4):250-278.

18. Dronge AS, Perkal MF, Kancir S, Concato J, Aslan M, Rosenthal RA. Long-term glycemic control and postoperative infectious complications. Arch Surg. 2006;141:375-380.

19. Lindstrom D, Sadr Azodi O, Wladis A, et al. Effects of a perioperative smoking cessation intervention on postoperative complications: a randomized trial. Ann Surg. 2008;248(5):739-745.

20. Lee I, Agarwal RK, Lee BY, Fishman NO, Umscheid CA. Systematic Review and Cost Analysis Comparing Use of Chlorhexidine with Use of Iodine for Preoperative Skin Antisepsis to Prevent Surgical Site Infection. Infect Control Hosp Epidemiol. 2010;31(12):1-12.

21. Swenson BR, Hedrick TL, Metzger R, Bonatti H, Pruett TL, Sawyer RG. Effects of preoperative skin preparation on postoperative wound infection rates: a prospective study of 3 skin preparation protocols. Infect Control Hosp Epidemiol. 2009;30(10):964-971.

22. Kurz A, Sessler DI, Lenhardt R. Perioperative normothermia to reduce the incidence of surgical-wound infection and shorten hospitalization. Study of Wound Infection and Temperature Group. N Engl J Med. 1996;334(19):1209-1215.

23. Walz JM, Paterson CA, Seligowski JM, Heard SO. Surgical site infection following bowel surgery: a retrospective analysis of 1446 patients. Arch Surg. 2006;141(10):1014-1018; discussion 1018.
24. Leeds IL, Wick EC, Melton GB. Does close temperature regulation affect surgical site infection rates? Adv Surg. 2014;48:65-76.

25. Melton GB, Vogel JD, Swenson BR, Remzi FH, Rothenberger DA, Wick EC. Continuous intraoperative temperature measurement and surgical site infection risk: analysis of anesthesia information system data in 1008 colorectal procedures. Ann Surg. 2013;258(4):606-613.

26. Stratchounski LS, Taylor EW, Dellinger EP, Pechere JC. Antibiotic policies in surgery: a consensus paper. Int J Antimicrob Agents. 2005;26(4): 312-322.

27. Stone HH, Hooper CA, Kolb LD, Geheber CE, Dawkins EJ. Antibiotic prophylaxis in gastric, biliary and colonic surgery. Ann Surg. 1976; 184(4):443-452.

28. Polk Jr HC, Lopez-Mayor JF. Postoperative wound infection: a prospective study of determinant factors and prevention. Surgery. 1969;66(1): 97-103.

29. Bratzler DW, Dellinger EP, Olsen KM, et al. Clinical practice guidelines for antimicrobial prophylaxis in surgery. Surg Infect. 2013;14(1): 73-156.

30. Steinberg JP, Braun BI, Hellinger WC, et al. Timing of antimicrobial prophylaxis and the risk of surgical site infections: results from the trial to reduce antimicrobial prophylaxis errors. Ann Surg. 2009;250(1): $10-16$.

31. Schweizer ML, Chiang H-Y, Septimus E, et al. Association of a bundled intervention with surgical site infections among patients undergoing cardiac, hip or knee surgery. JAMA. 2015;313(21):2162-2171.

32. Southworth PM. Infections and exposures: reported incidents associated with unsuccessful decontamination of reusable surgical instruments. J Hosp Infect. 2014;88:127-131.

33. Rutala WA, Weber DJ. Healthcare Infection Control Practices Advisory Committee (HICPAC). Guideline for Disinfection and Sterilization in Healthcare Facilities, 2008. Atlanta: Centers for Disease Control and Prevention; 2008.

34. Cheung RJ, Ortiz D, DiMarino AJJ. GI endoscopic reprocessing practices in the United States. Gastrointest Endosc. 1999;50(3):362-368.

35. Haley RW. The scientific basis for using surveillance and risk factor data to reduce nosocomial infection rates. J Hosp Infect. 1995; 30 Suppl:3-14.

36. Lawson EH, Zingmond DS, Hall BL, Louie R, Brook RH, Ko CY. Comparison between clinical registry and medicare claims data on the classification of hospital quality of surgical care. Ann Surg. 2015;261(2): 290-296.

37. Letourneau AR, Calderwood MS, Huang SS, Bratzler DW, MaA, YokoeDS. Harnessing claims to improve detection of surgical site infections following hysterectomy and colorectal surgery. Infect Control Hosp Epidemiol. 2013;34(12):1321-1323.

38. Calderwood MS, Ma A, Khan YM, et al. Use of medicare diagnosis and procedure codes to improve detection of surgical site infections following hip arthroplasty, knee arthroplasty, and vascular surgery. Infect Control Hosp Epidemiol. 2012;33(1):40-49.

39. Barie PS, Eachempati SR. Surgical site infections. Surg Clin North Am. 2005;85(6):1115-1135, viii-ix.

40. Kirby JP, Mazuski JE. Prevention of surgical site infection. Surg Clin North Am. 2009;89(2):365-389, viii.

41. Stevens DL, Bisno AL, Chambers HF, et al. Practice guidelines for the diagnosis and management of skin and soft tissue infections: 2014 update by the infectious diseases Society of America. Clin Infect Dis. 2014;59(2).

42. Centers for Disease Control and Prevention (CDC). Surgical Site Infection (SSI) Event. Atlanta: Centers for Disease Control and Prevention; 2015. Available from: http://www.cdc.gov/nhsn/PDFs/ pscManual/9pscSSIcurrent.pdf. Accessed June 15, 2015. 


\section{Publish your work in this journal}

Ambulatory Anesthesia is an international, peer reviewed, open access journal publishing articles that address all aspects of ambulatory anesthesia practice, in particular: anesthetic techniques, sedation and safety practices, pharmacokinetics, preoperative evaluation, analgesia interventions, regulatory and compliance issues, postoperative recovery,

Submit your manuscript here: http://www.dovepress.com/ambulatory-anesthesia-journal
Dovepress

patient satisfaction, administrative topics, and cost analysis themes. The manuscript management system is completely online and includes a very quick and fair peer review system, which is all easy to use. Visit $\mathrm{http}: / /$ www.dovepress.com/testimonials.php to read real quotes from published authors. 